\title{
Hybrid Nanocavity Resonant Enhancement of Color Center Emission in Diamond
}

\author{
Paul E. Barclay, ${ }^{1, *}$ Kai-Mei C. Fu, ${ }^{1, \dagger}$ Charles Santori, ${ }^{1}$ Andrei Faraon, ${ }^{1}$ and Raymond G. Beausoleil ${ }^{1}$ \\ ${ }^{1}$ Hewlett-Packard Laboratories, 1501 Page Mill Road, Palo Alto, California 94304, USA
}

(Received 20 June 2011; published 7 September 2011)

\begin{abstract}
Resonantly enhanced emission from the zero-phonon line of a diamond nitrogen-vacancy (NV) center in single crystal diamond is demonstrated experimentally using a hybrid whispering gallery mode nanocavity. A $900 \mathrm{~nm}$ diameter ring nanocavity formed from gallium phosphide, whose sidewalls extend into a diamond substrate, is tuned onto resonance at a low temperature with the zero-phonon line of a negatively charged NV center implanted near the diamond surface. When the nanocavity is on resonance, the zero-phonon line intensity is enhanced by approximately an order of magnitude, and the spontaneous emission lifetime of the $\mathrm{NV}$ is reduced by as much as $18 \%$, corresponding to a $6.3 \mathrm{X}$ enhancement of emission in the zero photon line.
\end{abstract}

Subject Areas: Nanophysics, Photonics, Quantum Information

The diamond nitrogen-vacancy (NV) center is an optically active impurity which combines many of the desirable properties of quantum dots and laser-trapped atoms. Optical transitions of diamond NV centers can display low inhomogeneous broadening, and have been used to generate single photons [1], manipulate single electron spins [2], and control nearby nuclear spin impurities [3-5]. Remarkably, the room temperature electron spin coherence times of NVs can exceed a millisecond [6]. These properties make NVs a promising qubit for proposed quantum networks [7], and an attractive system for applications such as magnetometry [8] and low power optical switching [9]. An outstanding challenge limiting the use of NV centers as qubits in quantum information processing is the creation of a platform for mediating interactions between them. A promising approach to this problem is to create an onchip quantum network in which NVs interact optically via nanophotonic interconnects [10]. Coupling NVs to optical cavities plays a crucial role in this implementation by enhancing the NV emission into a well-defined optical mode, which can be efficiently coupled to waveguides and routed on-chip. Cavity enhancement of the emission is particularly important for NV centers, as it provides a means for increasing the relative brightness of the narrowband zero-phonon line (ZPL) emission relative to the broadband phonon assisted emission. The selection of the

\footnotetext{
*Present address: Institute for Quantum Information Science, University of Calgary, Calgary, AB, T2N 1N3, Canada; NRCNational Institute for Nanotechnology, 11421 Saskatchewan Drive NW, Edmonton, AB T6G 2M9, Canada. pbarclay@ucalgary.ca

${ }^{\dagger}$ Present address: Department of Physics, University of Washington, 4014 University Way NE, Seattle, WA 98105, USA.

Published by the American Physical Society under the terms of the Creative Commons Attribution 3.0 License. Further distribution of this work must maintain attribution to the author(s) and the published article's title, journal citation, and DOI.
}

emission into the ZPL is necessary for protocols involving coherent interactions or indistinguishable photons.

Demonstrating diamond-based quantum networks has recently prompted significant research into integrating NV centers with photonic devices [11]. Efforts to efficiently couple NVs in nanocrystalline diamond to nanophotonic structures [12-15] have been limited by poor NV optical properties in nanocrystals compared to those found in single crystal diamond. Fabricating nanophotonic devices directly from single crystal diamond has recently made important progress $[11,16,17]$, limited primarily by fabrication difficulties related to creating thin films of single crystal diamond necessary for optical confinement in three dimensions. An alternative approach, which leverages existing semiconductor processing technology, is to create photonic structures from hybrid material systems in which a thin waveguiding layer is bonded to the surface of a single crystal diamond substrate. Light localized within waveguides and microcavities lithographically defined in the waveguiding layer can interact evanescently with NVs positioned near the surface of the diamond substrate; these hybrid devices are naturally suited for coupling to arrays of NV implanted near surfaces, such as those studied in Ref. [18]. The hybrid approach can take advantage of properties of the waveguiding material that are not available in all-diamond systems, for example, nonlinear or optoelectronic responses, which are useful for integrated optical modulation [19]. Hybrid semiconductor-diamond devices were used in Refs. [20,21] to demonstrate evanescent coupling between ensembles of NVs and micron-scale photonic waveguides and cavities. Here, we demonstrate optical coupling between a nanoscale hybrid optical cavity and a single diamond NV center, and measure resonant Purcell enhanced spontaneous emission into the ZPL.

The nanocavities studied here were realized from a hybrid geometry consisting of a gallium phosphide (GaP, $250 \mathrm{~nm}$ thickness, $n_{\mathrm{GaP}} \sim 3.3$ ) whispering gallery mode ring nanocavity supported by a single crystal diamond substrate. A typical device is shown in Fig. 1(a). They 
were fabricated following the process for hybrid microdisk cavities in Ref. [20]; no changes to account for the ring geometry of the nanocavities used here were needed. The diamond substrate consists of a chemical vapor deposition grown electronic grade single crystal diamond sample (Element Six) subjected to ion implantation $\left(\mathrm{N}^{+} 10 \mathrm{keV}\right.$, $\left.1 \times 10^{10} \mathrm{~cm}^{-2}\right)$ and annealing $\left(900^{\circ} \mathrm{C}\right.$ in $\left.\mathrm{H}_{2} / \mathrm{Ar}\right)$ to create $\mathrm{NVs}$ close to the diamond surface. A subsequent oxygen anneal step maximized the $\mathrm{NV}^{-} / \mathrm{NV}^{0}$ ratio near the surface [22]. The nanocavity diameter $d \sim 900 \mathrm{~nm}$ is 5 times smaller than in previous work [20]. To enhance optical confinement, the GaP sidewalls were extended $\sim 600 \mathrm{~nm}$ into the diamond using an oxygen plasma etch, decreasing the effective refractive index of the underlying nanocavity substrate.

This structure supports whispering gallery modes whose field is primarily confined inside the $\mathrm{GaP}$ and interacts evanescently with NVs near the diamond surface. Figures 1(b) and 1(c) show the simulated field profile of the whispering gallery mode supported by this structure with an azimuthal mode index $m=9$, fundamental radial order $(p=0)$, and TE-like polarization (where the dominant electric field component is radially polarized). The field profile was calculated using a finite difference
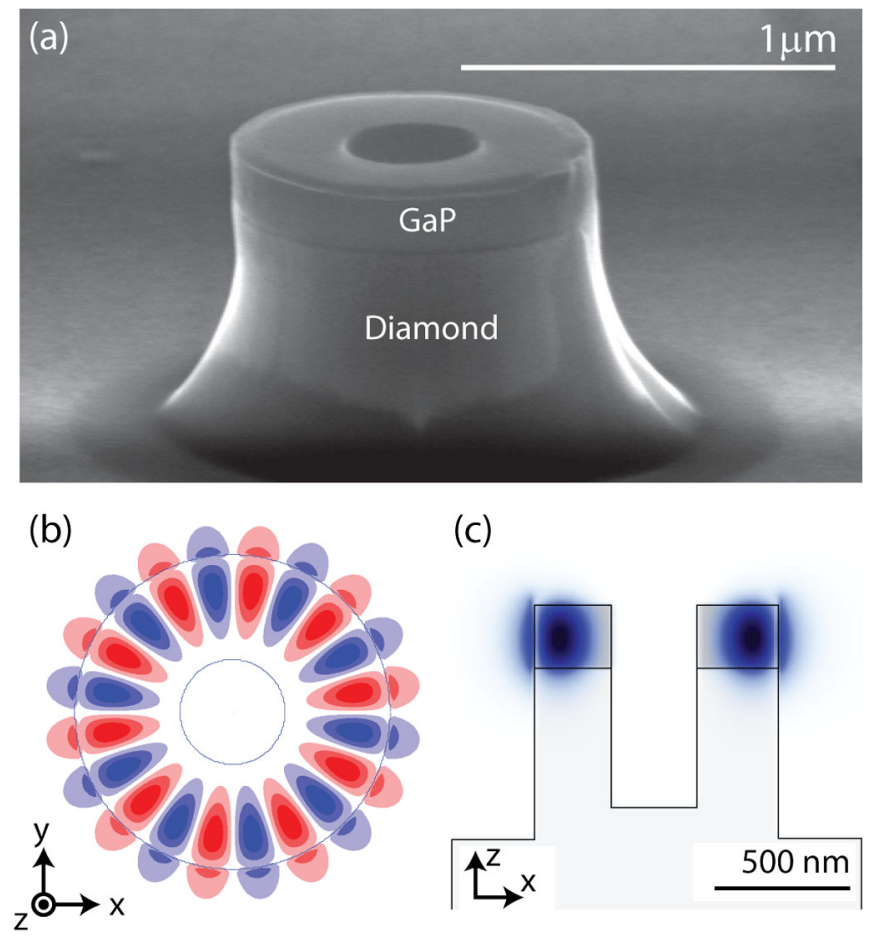

(c)

FIG. 1. (a) Scanning electron microscope image of a hybrid GaP-diamond whispering gallery mode nanocavity. (b) Top-view and (c) cross-section of the dominant electric field component $E_{r}$ of the lowest order TE-like standing-wave mode supported by the device in (a), with resonance wavelength $\lambda_{o} \sim 637 \mathrm{~nm}$. The fields are calculated using finite difference time domain simulations. The field in (b) is plotted in the $x-y$ center-plane of the $\mathrm{GaP}$ layer. time domain simulation (FDTD) [23], and has a resonance wavelength close to $\lambda_{\mathrm{ZPL}} \sim 637 \mathrm{~nm}$ of the $\mathrm{NV}^{-} \mathrm{ZPL}$, mode volume $V \sim 3.0\left(\lambda / n_{\mathrm{GaP}}\right)^{3}$, which is defined by the peak electric field energy density, and a maximum intensity in the diamond of $\eta_{\text {dia }} \sim 0.11$ of the peak intensity in the $\mathrm{GaP}$. The theoretical radiation limited quality factor $Q_{\text {rad }}$ for these structures exceeds $10^{6}$; in practice, fabrication imperfections and material absorption will limit $Q$ below $Q_{\text {rad }}[20]$.

The fraction of spontaneous emission radiated from an evanescently coupled NV into the nanocavity mode described above can be predicted by calculating the Purcell enhancement to the zero-phonon emission at $637 \mathrm{~nm}$. In bulk, a NV ${ }^{-}$color center radiates a fraction $\zeta_{\mathrm{ZPL}} \sim 3 \%$ of its emission into the ZPL, which is estimated from our measurements of the area under the ZPL in the spontaneous emission spectrum from an ensemble of NVs. In the presence of a resonant nanocavity, the emission into the ZPL is enhanced by a factor $F_{\mathrm{ZPL}}$ due to the Purcell effect [24,25]:

$$
F_{\mathrm{ZPL}}=\frac{3}{4 \pi^{2}} \frac{n_{o}}{n_{d}}\left(\frac{\lambda_{\mathrm{ZPL}}}{n_{\mathrm{GaP}}}\right)^{3} \frac{Q}{V}\left|\frac{\mu \cdot \mathbf{E}\left(\mathbf{r}_{\mathrm{NV}}\right)}{\mathbf{E}_{o}}\right|^{2},
$$

where $\mathbf{E}\left(\mathbf{r}_{\mathrm{NV}}\right)$ is the electric field strength at the NV, $\mathbf{E}_{o}$ is the peak nanocavity field strength, $\mu$ is a unit vector describing the NV dipole orientation, $n_{o}$ is the refractive index at the peak field location, and $n_{d}$ is the refractive index of diamond. The total cavity enhanced $\mathrm{NV}^{-}$spontaneous emission rate is then given by

$$
\gamma_{c} \sim \gamma_{o}\left(1+F_{\mathrm{ZPL}} \zeta_{\mathrm{ZPL}}\right)
$$

where $\gamma_{o}$ is the bulk $\mathrm{NV}^{-}$spontaneous emission rate. Equation (2) assumes that the NV-cavity system is in the "bad cavity" limit, and neglects the modification of off resonance NV emission by the nanocavity.

The nanocavity optical properties were studied by exciting the device with a $532 \mathrm{~nm}$ source and measuring the resulting photoluminescence (PL). A scanning confocal microscope (0.6 NA objective) was used to excite and collect PL from a submicron diameter spot on the nanocavity. Collected light was directed to a spectrometer or a time resolved photon counting module. For the implantation dose used here, the NV density is such that a small number $(\sim 1-10)$ of NVs were typically excited by the excitation spot. All of the measurements were performed with the sample mounted in a liquid helium flow cryostat and cooled to $6 \mathrm{~K}$. Figure 2(a) shows a broad wavelength, low resolution $(0.14 \mathrm{~nm})$ spectrum of the nanocavity studied here when it was excited with a clockwise $(\mathrm{CW})$ $532 \mathrm{~nm}$ source $(\sim 1 \mathrm{~mW})$. The emission from the $637 \mathrm{~nm}$ ZPL of negatively charged NVs is clearly visible. Peaks in the emission corresponding to PL coupled to nanocavity modes are also evident and include a TE mode bluedetuned $3 \mathrm{~nm}$ from the $\mathrm{NV}^{-} \mathrm{ZPL}$. The modal polarization labels indicated in Fig. 2(a) were determined by comparing 

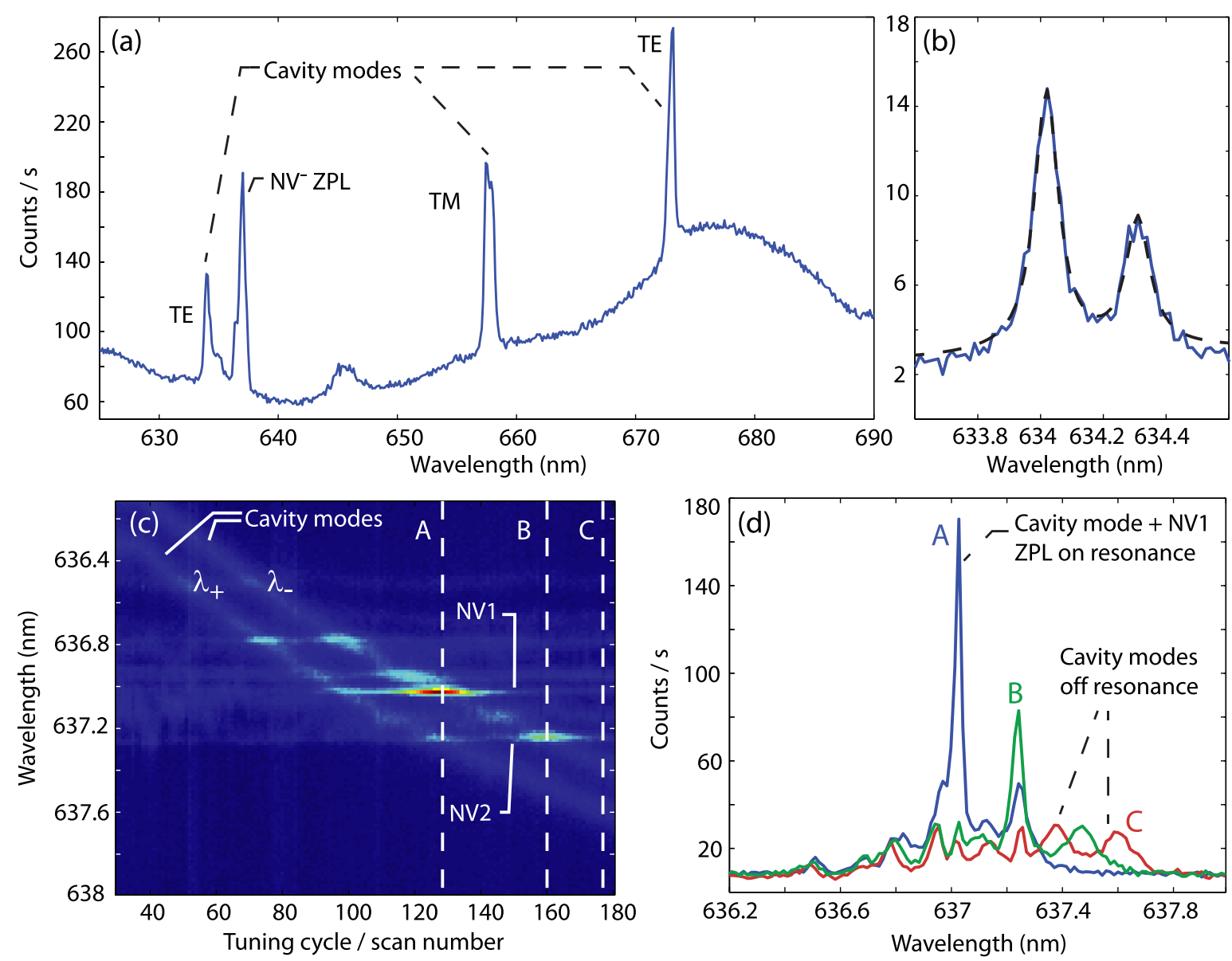

FIG. 2. (a) Spectrum of the nanocavity studied in (c) and (d) prior to tuning the nanocavity modes. (b) High-resolution PL spectrum of the nanocavity mode in (a) closest to the $\mathrm{NV}^{-}$ZPL. The dashed line is a fit to the data of two incoherently added Lorentizian lineshapes. (c) Nanocavity PL spectrum as a function of cavity tuning cycles. Each tuning cycle corresponds to releasing a fixed volume of Xe gas into the cryostat. (d) Nanocavity PL spectra when the nanocavity is on resonance with the ZPL of NV1 and NV2 (spectra A and B, respectively), and off resonance from any NV ZPL (spectra C). Spectra A, B, and C are measured after the tuning cycles indicated by the dashed lines in (c).

the resonance spacing with FDTD predicted values, as shown in Ref. [20], and by comparing their relative tuning rates in the measurements described below.

Figure 2(b) shows a high-resolution $(\sim 0.02 \mathrm{~nm})$ spectrum of PL from the nanocavity mode closest to the $\mathrm{NV}^{-}$ZPL. This reveals that the nanocavity mode has a doublet structure consisting of two peaks at wavelengths $\lambda_{ \pm}=\lambda_{o} \pm \Delta \lambda / 2$ with full-width at half-max $\delta \sim 94 \mathrm{pm}$ $\left(Q_{ \pm} \sim 6800\right) . \Delta \lambda=0.27 \mathrm{~nm}$ is the doublet splitting, and $\delta$ was determined by fitting the data with two incoherently superimposed Lorentzian lineshapes, as shown in Fig. 2(b). The doublet structure results from nanocavity surface roughness and imperfections that couple the nominally degenerate clockwise and counterclockwise circulating whispering gallery modes, creating nondegenerate standing-wave modes [26].

As described by Eq. (1), when a nanocavity mode is resonant with the NV ZPL, the NV spontaneous emission rate can be enhanced through the Purcell effect. We demonstrate this by tuning the nanocavity in Fig. 2(a) through resonance with the $\mathrm{NV}^{-} \mathrm{ZPL}$, and show that the nanocavity significantly enhances the ZPL intensity of coupled NVs. The nanocavity resonances are tuned by injecting Xe gas into the cryostat [27], where it condenses on the nanocavity surface and redshifts the wavelengths of the nanocavity modes. In Fig. 2(c), the PL spectra in the vicinity of the $\mathrm{NV}^{-} \mathrm{ZPL}$ are shown at discrete steps in the tuning process as the nanocavity mode closest to the $\mathrm{NV}^{-}$ ZPL is tuned from $636 \mathrm{~nm}$ to $637.5 \mathrm{~nm}$. The nanocavity doublet resonance shifts diagonally across Fig. 2(c) as the number of Xe tuning cycles increases. ZPL emission between $636.8 \mathrm{~nm}-637.4 \mathrm{~nm}$ from several distinct NVs is visible, creating horizontal features whose center wavelength is unaffected by the Xe tuning. The inhomogeneous distribution of ZPL wavelengths is possibly the result of residual strain from the implantation step or the diamond etching. In some cases, when the nanocavity modes cross the ZPL lines, the ZPL PL intensity increases. In particular, 
a large enhancement is visible when the $\lambda_{-}$nanocavity mode is resonant with a ZPL at $637.0 \mathrm{~nm}$ (labeled NV1). A comparatively modest enhancement is observed when $\lambda_{-}$ is resonant with a ZPL at $637.25 \mathrm{~nm}$ (labeled NV2). The relative magnitude of enhancement is shown in Fig. 2(d), which compares the PL spectrum when the nanocavity mode is on resonance with the NV1 ZPL [slice A in Fig. 2(c)], on resonance with the NV2 ZPL [slice B in Fig. 2(c)], and when it is detuned [slice $\mathrm{C}$ in Fig. 2(c)].

In general, the magnitude of the enhancement depends on the NV position and dipole orientation relative to the nanocavity field maximum, as indicated in Eq. (1). The nanocavity standing-wave whispering gallery modes have well-defined electric field amplitude nodes and antinodes, whose specific phase is determined by the nanocavity imperfections [26]. For maximum NV-cavity coupling, the NV must be located at an antinode of one of the two standing-wave modes, which is necessarily a node of the orthogonal standing-wave mode. This nanoscale sensitivity of the coupling strength on the NV position is illustrated in Fig. 2(c) by the asymmetry of the NV1 ZPL enhancement when the two nanocavity standing wave doublet peaks $\left(\lambda_{ \pm}\right)$are on resonance. The $\lambda_{-}$mode strongly enhances the NV1 ZPL PL intensity; the $\lambda_{+}$mode does not significantly affect the NV1 ZPL. This indicates that NV1 is positioned near an antinode of the $\lambda_{-}$nanocavity mode.

To quantitatively determine the degree of enhancement, we measure the effect of the nanocavity mode on the spontaneous emission lifetimes of NV1 and NV2. Figure 3(a) shows a time resolved PL when the device is excited with a pulsed green source $(4.75 \mathrm{MHz}$ repetition rate, $520 \mathrm{~nm}$ center wavelength, $28 \mathrm{~nm}$ bandwidth,
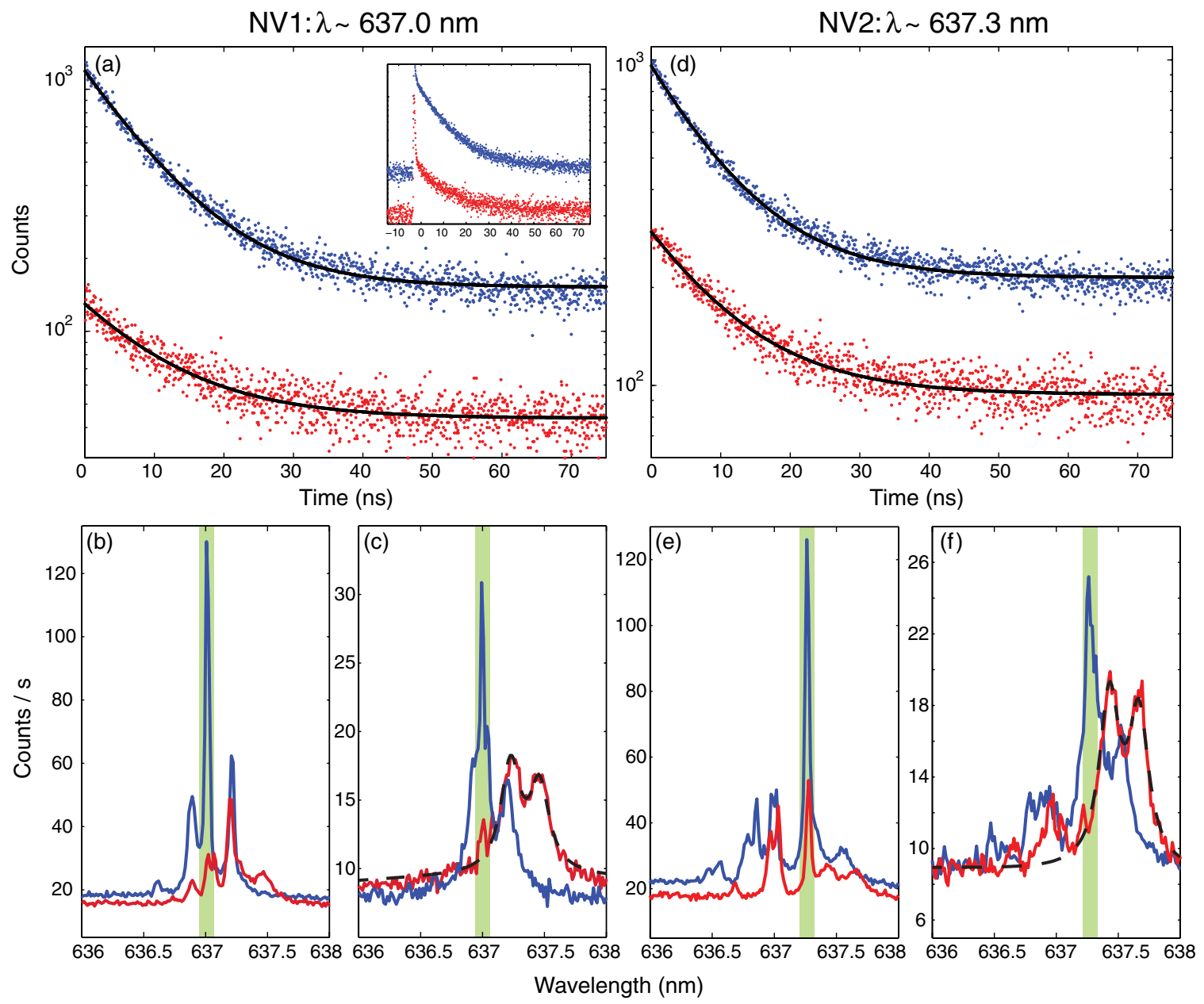

FIG. 3. (a) Time resolved photoluminescence of NV1 excited with a pulsed green source, when the NV1 ZPL is on (blue) and off (red) resonance with the nanocavity $\lambda_{-}$mode. The time origin is chosen $\sim 3.0 \mathrm{~ns}$ after the excitation pulse peak (see inset) so that fast decaying nanocavity background does not influence the fits (solid lines). Nanocavity spectra under (b) CW and (c) pulsed 532 nm excitation, when NV1 ZPL is on (blue) and off (red) resonance with the nanocavity mode. The green shaded regions indicate the monochromator spectral window used for the lifetime measurements in (a). The dashed line in (c) is a fit to the data consisting of two incoherently superimposed Lorentzian lineshapes. (d)-(f) Data analogous to (a)-(c), with the nanocavity $\lambda_{-}$mode tuned on and off resonance with the NV2 ZPL. 
$300 \mu \mathrm{W}$ average power), with the nanocavity $\lambda_{-}$mode tuned on and off resonance with NV1. To ensure that the spontaneous emission properties of only the NV of interest were being measured, the PL was spectrally filtered using a monochromator centered at the wavelength of the NV1 ZPL (637.0 nm). This emission was then directed to a timecorrelated single photon counting module that records the photon detection time relative to the excitation pulse.

Figures 3(b) and 3(c) show the spectra obtained for the on and off resonance measurements of NV1 under CW and pulsed excitation, respectively. In both cases an enhancement to the NV1 ZPL intensity is observed when the nanocavity is on resonance. The non-NV related emission from the nanocavity modes is strong in the pulsed excitation spectra [Fig. 3(c)] due to the emission related to the nanocavity material or fabrication residue. This emission decays quickly $(\sim 3 \mathrm{~ns})$ compared to the $\mathrm{NV}$ emission, which is shown by the inset to Fig. 3(a). Figure 3(c) also reveals that the nanocavity $Q$ and doublet splitting was modified compared to the measurements in Fig. 2. During the lifetime measurements, $Q \sim 3000$ and $\Delta \lambda \sim 0.22 \mathrm{~nm}$, which was determined from the fit in Fig. 3(c). This degradation in $Q$, compared to the value observed during the measurements in Fig. 2, may be related to repeated condensation and evaporation of Xe or other cyrostat contaminants over the course of several measurement cycles for this device. Local modification of the GaP properties by the green excitation laser, as reported in Ref. [15], may also affect the device characteristics.

The NV1 spontaneous emission rate can be determined by fitting the data in Fig. 3(a) with simple exponential decay functions. These fits indicate that the NV1 spontaneous emission lifetime is $\tau_{c}=9.7 \pm 0.07 \mathrm{~ns}$ and $\tau_{o}=$ $11.6 \pm 0.3 \mathrm{~ns}$ when the nanocavity mode is on and off resonance, respectively, with the NV1 ZPL. This indicates that the nanocavity enhancement of the ZPL emission appreciably modifies the total spontaneous emission rate of the NV. From $\gamma_{o, c}=1 / \tau_{o, c}$ and the lifetime fits extracted from Fig. 3, we estimate $F_{\mathrm{ZPL}} \sim 6.3 \pm 1.0$. This corresponds to enhancing the fraction of NV1 emission into the ZPL from $\zeta_{\text {ZPL }} \sim 3 \%$ to $\sim 16 \%$.

The effect of the nanocavity on the NV2 ZPL is illustrated in Fig. 3(d)-3(f), which shows lifetime and spectral data when the nanocavity $\lambda_{-}$mode is tuned on and off resonance. These measurements were performed in the same manner as the NV1 measurements, but with the monochromator centered at the NV2 ZPL $(637.2 \mathrm{~nm})$. Note that during these measurements, the excitation spot alignment was optimized to maximize the NV2 ZPL emission, resulting in larger on and off resonance contrast in Fig. 3(e) compared to Fig. 2(c). Fits to the data in Fig. 3(d) indicate that the NV2 spontaneous emission lifetime is $\tau_{c}=9.84 \pm 0.08 \mathrm{~ns}$ and $\tau_{o}=$ $11.0 \pm 0.2 \mathrm{~ns}$ when the nanocavity mode is on and off resonance, respectively, with the NV2 ZPL. This indicates that $F_{\mathrm{ZPL}} \sim 3.8 \pm 0.7$ for NV2.
From Eq. (1), the FDTD predicted mode volume and field distribution presented above, and $Q \sim 3000$ measured during the lifetime measurement, the maximum expected $F_{\mathrm{ZPL}}$ can be calculated. For a NV optimally positioned relative to the nanocavity studied here, the ZPL emission would be enhanced by $F_{\mathrm{ZPL}}^{0 \mathrm{~nm}}=12$ and $F_{\mathrm{ZPL}}^{20 \mathrm{~nm}}=6.7$, assuming the $\mathrm{NV}$ is positioned at the surface and $20 \mathrm{~nm}$ below the surface (the maximum expected implantation depth), respectively. $F_{\mathrm{ZPL}}$ becomes smaller with the $\mathrm{NV}$ distance to the surface due to the evanescent decay of the nanocavity mode electric field intensity, which scales roughly as $\exp \left(-2 \kappa_{z} z\right)$, where $\kappa_{z}=(2 \pi / \lambda) \sqrt{n_{\mathrm{GaP}}^{2}-n_{\mathrm{dia}}^{2}} \cdot F_{\mathrm{ZPL}}$ is also reduced when the $\mathrm{NV}$ is not positioned at an antinode of the standing-wave mode, and if the NV dipole is misaligned relative to the field polarization. As discussed above, the relative degree of ZPL enhancement when it is resonant with the $\lambda_{-}$and $\lambda_{+}$modes in Fig. 2(a) suggests that NV1 is closely positioned near a node of the $\lambda_{+}$standing wave, and an antinode of the $\lambda_{-}$ standing wave. Note that if the standing-wave mode splitting were small compared to the cavity mode linewidth, the mode antinodes would self-align with an on-resonance NV. Reaching this regime may be possible by reducing device imperfections through improved fabrication. Alternatively, tuning the standing-wave mode orientation using an externally controlled perturbation may be possible [28].

In future work, $F_{\mathrm{ZPL}}$ can be increased using higher $Q$ nanocavities and by further reducing $V$. Additional studies are required to determine the mechanism limiting the $Q$ of the devices studied here, as well as the degradation in $Q$ observed over the course of the measurements. Surface roughness and other defects are visible in the scanning electron microscope image in Fig. 1(a), and the large mode splitting $\Delta \lambda$ indicates that surface scattering is present in these devices and likely plays an important role in limiting $Q$. By eliminating this roughness, it should be possible to achieve $Q$ in excess of $2 \times 10^{4}$, as was demonstrated in larger diameter hybrid GaP-diamond devices [20]. Achieving this $Q$ with the specific coupled $\mathrm{NV}$-nanocavity device studied here would increase $F_{\text {ZPL }}$ to $\sim 42$, which corresponds to enhancing the ZPL branching ratio to $\zeta_{\mathrm{ZPL}} \sim 57 \%$.

Shrinking $V$ is immediately possible by coupling a NV to the TM mode ( $m=10, p=0)$ of the nanocavity studied here. The TM mode has a smaller mode volume, $V \sim 2.6\left(\lambda / n_{\mathrm{GaP}}\right)^{3}$, than the TE mode, and a larger relative intensity at the diamond surface, $\eta_{\text {dia }} \sim 0.15$, resulting in an approximately $52 \%$ increase in $F_{\mathrm{ZPL}}^{0 \mathrm{~nm}}$ compared to the TE mode for a given $Q$ and an optimally positioned NV. Further reduction in $V$ is possible by decreasing the nanocavity diameter to $d=650 \mathrm{~nm}$; the resulting device supports a TM mode with $V \sim 1.7\left(\lambda / n_{\mathrm{GaP}}\right)^{3}$ and $\eta_{\text {dia }} \sim 0.39$ and radiation $Q_{\text {rad }} \sim 4 \times 10^{4}$. Ultrasmall $V$ is possible using a hybrid GaP-diamond photonic crystal nanocavity proposed in Ref. [29], which supports TE modes with $V \sim 0.5\left(\lambda / n_{\mathrm{GaP}}\right)^{3}$ while maintaining a radiation limited 
$Q_{\text {rad }}>10^{6}$. These photonic crystal nanocavities are naturally suited to being interconnected, and are a promising system for implementing an on-chip quantum network to enable interactions between NVs.

In the immediate future, ZPL photon detection rates exceeding those that can be achieved by using a microscope objective to collect $\mathrm{NV}$ emission can be realized by combining the Purcell enhancement from the ring nanocavites demonstrated here, and the fiber taper waveguide PL collection studied in Ref. [30]. This would be an important tool for creating quantum information resources such as NV-photon entanglement [31], which rely on narrowband measurement of the ZPL emission. More generally, a fiber coupled nanocavity-NV system could be used as a bright narrowband source of indistinguishable photons, and to improve readout rates of the NV spin properties.

This material is based upon work supported by the Defense Advanced Research Projects Agency under Contract No. HR0011-09-1-0006 and the Regents of the University of California.

[1] C. Kurtsiefer, S. Mayer, P. Zarda, and H. Weinfurter, Stable Solid-state Source of Single Photons, Phys. Rev. Lett. 85, 290 (2000).

[2] F. Jelezko, T. Gaebel, I. Popa, A. Gruber, and J. Wrachtrup, Observation of Coherent Oscillations in a Single Electron Spin, Phys. Rev. Lett. 92, 076401 (2004).

[3] F. Jelezko, T. Gaebel, I. Popa, M. Domhan, A. Gruber, and J. Wrachtrup, Observation of Coherent Oscillation of a Single Nuclear Spin and Realization of a Two-Qubit Conditional Quantum Gate, Phys. Rev. Lett. 93, 130501 (2004).

[4] L. Childress, M. V. Gurudev Dutt, J. M. Taylor, A. S. Zibrov, F. Jelezko, J. Wrachtrup, P. R. Hemmer, and M. D. Lukin, Coherent Dynamics of Coupled Electron and Nuclear Spin Qubits in Diamond, Science 314, 281 (2006).

[5] M. V. Gurudev Dutt, L. Childress, L. Jiang, E. Togan, J. Maze, F. Jelezko, A. S. Zibrov, P. R. Hemmer, and M. D. Lukin, Quantum Register Based on Individual Electronic and Nuclear Spin Qubits in Diamond, Science 316, 1312 (2007).

[6] G. Balasubramanian, P. Neumann, D. Twitchen, M. Markham, R. Kolesov, N. Mizuochi, J. Isoya, J. Achard, J. Beck, J. Tissler, V. Jacques, P. Hemmer, F. Jelezko, and F. Wrachtrup, Ultralong Spin Coherence Time in Isotopically Engineered Diamond, Nature Mater. 8, 383 (2009).

[7] H. Kimble, The Quantum Internet, Nature (London) 453, 1023 (2008).

[8] J. R. Maze, P. L. Stanwix, J. S. Hodges, S. Hong, J. M. Taylor, P. Cappellaro, L. Jiang, M. V. G. Dutt, E. Togan, A. S. Zibrov, A. Yacoby, R. L. Walsworth, and M. D. Lukin, Nanoscale Magnetic Sensing with an Individual
Electronic Spin in Diamond, Nature (London) 455, 644 (2008).

[9] H. Mabuchi, Cavity-QED Models of Switches for Attojoule-Scale Nanophotonic Logic, Phys. Rev. A 80, 45802 (2009).

[10] S. C. Benjamin, B. W. Lovett, and J. M. Smith, Prospects for Measurement-Based Quantum Computing with Solid State Spins, Laser Photonics Rev. 3, 556 (2009).

[11] I. Aharonovich, A. Greentree, and S. Prawer, Diamond Photonics, Nat. Photon. 5, 397 (2011).

[12] P.E. Barclay, O. Painter, C. Santori, K.-M. Fu, and R. Beausoleil, Coherent Interference Effects in a NanoAssembled Optical Cavity-QED System, Opt. Express 17, 8081 (2009).

[13] D. Englund, B. Shields, K. Rivoire, F. Hatami, J. Vuckovic, H. Park, and M.D. Lukin, Deterministic Coupling of a Single Nitrogen Vacancy Center to a Photonic Crystal Cavity, Nano Lett. 10, 3922 (2010).

[14] J. Wolters, A.W. Schell, G. Kewes, N. Nüsse, M. Schoengen, H. Döscher, T. Hannappel, B. Löchel, M. Barth, and O. Benson, Enhancement of the Zero Phonon Line Emission from a Single Nitrogen Vacancy Center in a Nanodiamond via Coupling to a Photonic Crystal Cavity, Appl. Phys. Lett. 97, 141108 (2010).

[15] T. van der Sar, J. Hagemeier, W. Pfaff, E. C. Heeres, S. M. Thon, H. Kim, P. M. Petroff, T.H. Oosterkamp, D. Bouwmeester, and R. Hanson, Deterministic Nanoassembly of a Coupled Quantum Emitter-Photonic Crystal Cavity System, Appl. Phys. Lett. 98, 193103 (2011).

[16] T. Babinec, B. Hausmann, M. Khan, Y. Zhang, J. Maze, P. Hemmer, and M. Loncar, A Diamond Nanowire SinglePhoton Source., Nature Nanotech. 5, 195 (2010).

[17] A. Faraon, P. E. Barclay, C. Santori, K.-M. C. Fu, and R. G. Beausoleil, Resonant Enhancement of the Zero-Phonon Emission from a Color Center in a Diamond Cavity, Nat. Photon. 5, 301 (2011).

[18] D. M. Toyli, C.D. Weis, G. D. Fuchs, T. Schenkel, and D. D. Awschalom, Chip-Scale Nanofabrication of Single Spins and Spin Arrays in Diamond, Nano Lett. 10, 3168 (2010).

[19] A. Yariv and P. Yeh, Photonics: Optical Electronics in Modern Communications (Oxford University Press, New York, 2006).

[20] P.E. Barclay, K.-M. Fu, C. Santori, and R. Beausoleil, Chip-Based Microcavities Coupled to NV Centers in Single Crystal Diamond, Appl. Phys. Lett. 95, 191115 (2009).

[21] K.-M. C. Fu, C. Santori, P.E. Barclay, I. Aharonovich, S. Prawer, N. Meyer, A. M. Holm, and R. G. Beausoleil, Coupling of Nitrogen-Vacancy Centers in Diamond to a GaP Waveguide, Appl. Phys. Lett. 93, 234107 (2008).

[22] K. Fu, C. Santori, P. Barclay, and R. Beausoleil, Conversion of Neutral Nitrogen-Vacancy Centers to Negatively Charged Nitrogen-Vacancy Centers Through Selective Oxidation, Appl. Phys. Lett. 96, 121907 (2010).

[23] A. F. Oskooi, D. Roundy, M. Ibanescu, P. Bermel, J. Joannopoulos, and S. G. Johnson, MEEP: A Flexible FreeSoftware Package for Electromagnetic Simulations by the FDTD Method, Comput. Phys. Commun. 181, 687 (2010). 
[24] E. M. Purcell, Spontaneous Emission Probabilities at Radio Frequencies, Phys. Rev. 69, 681 (1946).

[25] C. Santori, P. E. Barclay, K.-M. C. Fu, R. G. Beausoleil, S. Spillane, and M. Fisch, Nanophotonics for Quantum Optics using Nitrogen-Vacancy Centers in Diamond, Nanotechnology 21, 274008 (2010).

[26] M. Borselli, T. J. Johnson, and O. Painter, Beyond the Rayleigh Scattering Limit in High-Q Silicon Microdisks: Theory and Experiment, Opt. Express 13, 1515 (2005).

[27] S. Mosor, J. Hendrickson, B. C. Richards, J. Sweet, G. Khitrova, H. M. Gibbs, T. Yoshie, A. Scherer, O. B. Shchekin, and D.G. Deppe, Scanning a Photonic Crystal Slab Nanocavity by Condensation of Xenon, Appl. Phys. Lett. 87, 141105 (2005).

[28] A. Mazzei, S. Götzinger, L. de S. Menezes, G. Zumofen, O. Benson, and V. Sandoghdar, Controlled Coupling of Counterpropagating Whispering-Gallery Modes by a
Single Rayleigh Scatterer: A Classical Problem in a Quantum Optical Light, Phys. Rev. Lett. 99, 173603 (2007).

[29] P. E. Barclay, K. M. Fu, C. Santori, and R. G. Beausoleil, Hybrid Photonic Crystal Cavity and Waveguide for Coupling to Diamond NV-Centers, Opt. Express 17, 9588 (2009).

[30] K.-M. C. Fu, P. E. Barclay, C. Santori, A. Faraon, and R. G. Beausoleil, Low-Temperature Tapered-Fiber Probing of Diamond NV Ensembles Coupled to GaP Microcavities, New J. Phys. 13, 055023 (2011).

[31] E. Togan, Y. Chu, A.S. Trifonov, L. Jiang, J. Maze, M. V.G.D.L. Childress, A.S. Sørensen, P. R. Hemmer, A. S. Zibrov, and M. D. Lukin, Quantum Entanglement Between an Optical Photon and a Solid-State Spin Qubit, Nature (London) 466, 730 (2010). 